Claremont Colleges

Scholarship@ Claremont

All HMC Faculty Publications and Research

HMC Faculty Scholarship

8-1-2010

\title{
Combinatorial Trigonometry with Chebyshev Polynomials
}

Arthur T. Benjamin

Harvey Mudd College

Larry Ericksen

Pallavi Jayawant

Bates College

Mark Shattuck

University of Tennessee - Knoxville

\section{Recommended Citation}

Benjamin, A.T., Ericksen, L., Jayawant, P., \& Shattuck, M. (2010). Combinatorial trigonometry with Chebyshev polynomials. Journal of Statistical Planning and Inference, 140(8): 2157-2160. DOI: 10.1016/j.jspi.2010.01.011.

This Article is brought to you for free and open access by the HMC Faculty Scholarship at Scholarship @ Claremont. It has been accepted for inclusion in All HMC Faculty Publications and Research by an authorized administrator of Scholarship @ Claremont. For more information, please contact scholarship@cuc.claremont.edu. 
Provided for non-commercial research and education use. Not for reproduction, distribution or commercial use.

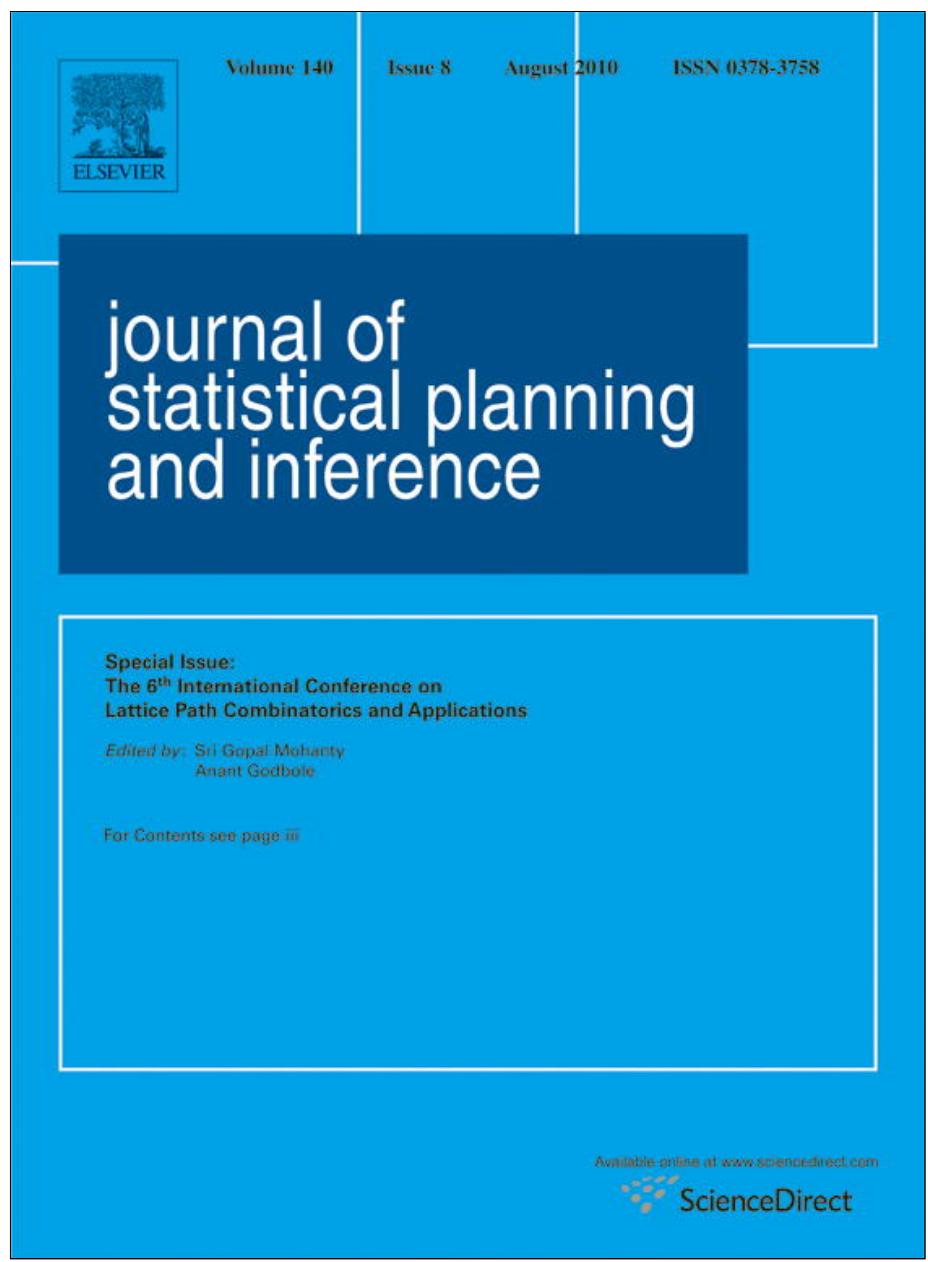

This article appeared in a journal published by Elsevier. The attached copy is furnished to the author for internal non-commercial research and education use, including for instruction at the authors institution and sharing with colleagues.

Other uses, including reproduction and distribution, or selling or licensing copies, or posting to personal, institutional or third party websites are prohibited.

In most cases authors are permitted to post their version of the article (e.g. in Word or Tex form) to their personal website or institutional repository. Authors requiring further information regarding Elsevier's archiving and manuscript policies are encouraged to visit:

http://www.elsevier.com/copyright 


\title{
Combinatorial trigonometry with Chebyshev polynomials
}

\author{
Arthur T. Benjamin ${ }^{\mathrm{a}, *}$, Larry Ericksen ${ }^{\mathrm{b}}$, Pallavi Jayawant ${ }^{\mathrm{c}}$, Mark Shattuck ${ }^{\mathrm{d}}$ \\ a Department of Mathematics, Harvey Mudd College, Claremont, CA 91711, USA \\ ${ }^{\mathrm{b}}$ P.O. Box 172, Millville, NJ 08332, CA, USA \\ ${ }^{\mathrm{c}}$ Department of Mathematics, Bates College, Lewiston, ME 04240-6028, USA \\ d Department of Mathematics, University of Tennessee, Knoxville, TN 37006-1300, USA
}

\section{A R T I C L E I N F O}

Available online 20 January 2010

Keywords:

Chebyshev polynomials

Combinatorial proof

Tiling

\begin{abstract}
A B S T R A C T
We provide a combinatorial proof of the trigonometric identity $\cos (n \theta)=T_{n}(\cos \theta)$, where $T_{n}$ is the Chebyshev polynomial of the first kind. We also provide combinatorial proofs of other trigonometric identities, including those involving Chebyshev polynomials of the second kind.
\end{abstract}

(c) 2010 Elsevier B.V. All rights reserved.

\section{Introduction}

The Chebyshev polynomials have many beautiful properties and countless applications, arising in a variety of continuous settings. They are a sequence of orthogonal polynomials appearing in approximation theory, numerical integration, and differential equations. In this paper we approach them instead as discrete objects, counting the sum of weighted tilings. Using this combinatorial approach, one can prove numerous identities, as is done in Benjamin and Walton (2009), Benjamin and Walton (2010), Shapiro (1981), and Walton (2007). In this note we provide a combinatorial proof of perhaps the most fundamental of Chebyshev properties, namely the trigonometric identity

$$
\cos (n \theta)=T_{n}(\cos \theta)
$$

where $T_{n}$ is the Chebyshev polynomial of the first kind. We also provide combinatorial proofs of other trigonometric identities, including those involving Chebyshev polynomials of the second kind.

The Chebyshev polynomials of the first kind are defined by $T_{0}(x)=1, T_{1}(x)=x$, and for $n \geq 2$,

$$
T_{n}(x)=2 x T_{n-1}(x)-T_{n-2}(x) .
$$

The next few polynomials are $T_{2}(x)=2 x^{2}-1, T_{3}(x)=4 x^{3}-3 x, T_{4}(x)=8 x^{4}-8 x^{2}+1, T_{5}(x)=16 x^{5}-20 x^{3}+5 x$.

The Chebyshev polynomials of the second kind differ only in the initial conditions. They are defined by $U_{0}(x)=1$, $U_{1}(x)=2 x$, and for $n \geq 2$,

$$
U_{n}(x)=2 x U_{n-1}(x)-U_{n-2}(x) .
$$

The next few polynomials are $U_{2}(x)=4 x^{2}-1, U_{3}(x)=8 x^{3}-4 x, U_{4}(x)=16 x^{4}-12 x^{2}+1, U_{5}(x)=32 x^{5}-32 x^{3}+6 x$.

\footnotetext{
* Corresponding author.

E-mail addresses: benjamin@hmc.edu (A.T. Benjamin), LE22@cornell.edu (L. Ericksen).
} 


\section{Chebyshev polynomials of the first kind}

Chebyshev polynomials have a simple combinatorial interpretation, which were first explored by Shapiro (1981). We define an $n$-tiling to be a sequence of squares (of length one) and dominoes (of length two) with a total length of $n$. For example, there are exactly five 4-tilings, namely $s s s s, s s D, s D s, D s s$, and $D D$, where $s$ denotes a square and $D$ denotes a domino. For Chebyshev polynomials of the first kind, we assign each of our square tiles, with one possible exception, a weight of $2 x$, and each domino is assigned a weight of -1 . The exception occurs if a square occurs as the first tile in the tiling, and here we assign that initial square a weight of $x$, but all subsequent squares still get a weight of $2 x$. We define the weight of the tiling to be the product of the weights of its tiles. For example, the aforementioned 4-tilings, ssss, ssD, sDs, Dss, and $D D$, have respective weights $8 x^{4},-2 x^{2},-2 x^{2},-4 x^{2}$, and 1 . Note that the sum of the weights of all 4-tilings is $8 x^{4}-8 x^{2}+1$, which is equal to $T_{4}(x)$.

In general, a simple induction argument on $n$ reveals (or see Benjamin and Quinn, 2003, Chapter 3) the following theorem.

Theorem 1. For $n \geq 0, T_{n}(x)$ is the sum of the weights of $n$-tilings where dominoes have weight -1 and squares have weight $2 x$, except for a square at the beginning of the tiling, which has weight $x$.

Our first trigonometric identity concerns the case where $x=\cos \theta$. Since $2 \cos \theta=e^{i \theta}+e^{-i \theta}$, we can refine the previous theorem. Here, we allow squares to come in two colors, white and black, where, except for an initial square, white squares have weight $e^{i \theta}$, and black squares have weight $e^{-i \theta}$. An initial square has half the weight given above, namely $\frac{1}{2} e^{i \theta}$ or $\frac{1}{2} e^{-i \theta}$, and all dominoes still have weight -1 . For example, if we let $D, w$, and $b$, respectively, denote a domino or white square or black square, then the 13-tiling $b b b D w b w w D D$ would have weight

$$
\frac{1}{2} e^{-i \theta} e^{-i \theta} e^{-i \theta}(-1) e^{i \theta} e^{-i \theta} e^{i \theta} e^{i \theta}(-1)(-1)=-\frac{1}{2} e^{-i \theta} .
$$

In general, we have:

Corollary 2. For $n \geq 0, T_{n}(\cos \theta)$ is the sum of the weights of $n$-tilings with dominoes, white squares, and black squares with weights described above.

We are now ready to establish our first theorem of combinatorial trigonometry.

Identity 1. For $n \geq 0, T_{n}(\cos \theta)=\cos (n \theta)$.

Proof. Observe that the $n$-tiling consisting of all white squares has weight $\frac{1}{2} e^{\text {in } \theta}$ and that the $n$-tiling with all black squares has weight $\frac{1}{2} e^{-i n \theta}$, and so the sum of their weights is $\left(e^{i n \theta}+e^{-i n \theta}\right) / 2=\cos (n \theta)$. The identity is proved by showing that the sum of all the other $n$-tilings is zero.

Let $X$ be an arbitrary $n$-tiling, and let $j$ be the first cell of the tiling such that cells $j$ and $j+1$ are occupied by a domino or cells $j$ and $j+1$ are occupied by squares of opposite colors. We consider two cases.

Case I: $j>1$. Suppose that $X$ has squares of opposite color on cells $j$ and $j+1$. Then let $X^{\prime}$ be the tiling obtained by replacing these two squares with a domino (and leaving the rest of the tiling unchanged). Alternatively, if $X$ has a domino on cells $j$ and $j+1$, then we obtain $X^{\prime}$ by replacing that domino with two squares of opposite color, where the square on cell $j$ has the same color as the square on cell $j-1$. Note that this rule is reversible: $\left(X^{\prime}\right)^{\prime}=X$. Since two squares of opposite color multiply to a weight of $e^{i \theta} e^{-i \theta}=1$ and dominoes have weight -1 , then $X$ and $X^{\prime}$ have weights that are opposite in sign and so the weights sum to zero.

Case II: $j=1$. Here $X$ must be of the form $w b Y$ or $b w Y$ or $D Y$, where $Y$ is an (n-2)- tiling. But since an initial $w b$ contributes a weight of $\frac{1}{2}$ and an initial $b w$ contributes a weight of $\frac{1}{2}$ and an initial domino contributes a weight of -1 , then it is clear that the weights of $w b Y, b w Y$, and $D Y$ must sum to zero.

We note that using the same logic, and replacing $2 \cos \theta$ with $z+1 / z$ (as in Ericksen, 2008, 2010), gives us

Identity 2. For $n \geq 0$, and any real or complex number $z$,

$$
T_{n}\left(\frac{z+\frac{1}{z}}{2}\right)=\frac{z^{n}+\frac{1}{z^{n}}}{2}
$$

It should be noted that this identity can be proved directly by a simple induction argument.

Another immediate corollary to Identity 1 , is the well-known composition theorem for Chebyshev polynomials.

Identity 3. For $m, n \geq 0, T_{m}\left(T_{n}(x)\right)=T_{m n}(x)$.

Proof. When $x=\cos \theta$,

$$
T_{m}\left(T_{n}(\cos \theta)\right)=T_{m}(\cos n \theta)=\cos (m n \theta)=T_{m n}(\cos \theta) .
$$


Since $T_{m}\left(T_{n}(x)\right)$ and $T_{m n}(x)$ are polynomials that agree for an infinite number of values of $x$, then they must be equal for all $x$.

The form of this theorem suggests that there may also be a bijective proof between mn-tilings and something like $m$-tilings of $n$-tilings. Such a bijection is presented in Benjamin and Walton (2009) and Walton (2007).

\section{Chebyshev polynomials of the second kind}

Due to its ideal initial conditions $U_{0}(x)=1$ and $U_{1}(x)=2 x$, Chebyshev polynomials of the second kind have a simpler combinatorial interpretation (Benjamin and Quinn, 2003) where all squares are given the same weight, including a square that occurs as the initial tile.

For example, the five 4-tilings $s s s s, s s D, s D s, D s s$, and $D D$ have respective weights $16 x^{4},-4 x^{2},-4 x^{2},-4 x^{2}$, and 1 , with a total weight of $16 x^{4}-12 x^{2}+1=U_{4}(x)$. And in general,

Theorem 3. For $n \geq 0, U_{n}(x)$ is the sum of the weights of $n$-tilings where dominoes have weight -1 and squares have weight $2 x$.

Likewise, by giving all white squares a weight of $e^{i \theta}$, all black squares a weight of $e^{-i \theta}$, and all dominoes a weight of -1 , we have

Corollary 4. For $n \geq 0, U_{n}(\cos \theta)$ is the sum of the weights of $n$-tilings with dominoes, white squares, and black squares with weights described above.

Using ideas from the proof of Identity 1, it is easy to show:

Identity 4. For $n \geq 0$,

$$
U_{n}(\cos \theta)=\frac{\sin ((n+1) \theta)}{\sin \theta}
$$

Proof. Equivalently, we prove $(\sin \theta) U_{n}(\cos \theta)=\sin ((n+1) \theta)$. Here we exploit the fact that $\sin \theta=\left(e^{i \theta}-e^{-i \theta}\right) / 2 i$. Hence the left side of our identity is the sum of the weights of all $(n+1)$-tilings, where the first tile is either a white square with weight $e^{i \theta} / 2 i$ or a black square with weight $-e^{-i \theta} / 2 i$, and is followed by an $n$-tiling where all white squares, black squares, and dominoes, have respective weights $e^{i \theta}, e^{-i \theta}$, and -1 .

As in the proof of Identity 1 , let $X$ be an $(n+1)$-tiling that does not consist of all white squares or all black squares. We let $j$ denote the first cell of $X$ such that cells $j$ and $j+1$ contain either two squares of opposite color or a domino. When $j>1$, we pair up $X$ with $X^{\prime}$ as before. If $j=1$, then $X$ must be of the form $X=w b Y$ or $X=b w Y$, where $Y$ is an (n-1)-tiling. But since an initial $w b$ contributes a weight of $\left(e^{i \theta} / 2 i\right) e^{-i \theta}=1 / 2 i$ and an initial $b w$ contributes a weight of $\left(-e^{-i \theta} / 2 i\right) e^{i \theta}=-1 / 2 i$ then the weights of $w b Y$ and $b w Y$ must sum to zero.

Hence the only $(n+1)$-tilings that are not canceled out are $w^{n+1}$ with weight $\left(e^{i \theta} / 2 i\right) e^{i n \theta}=e^{i(n+1) \theta} / 2 i$ and $b^{n+1}$ with weight $\left(-e^{-i \theta} / 2 i\right) e^{-i n \theta}=-e^{-i(n+1) \theta} / 2 i$. The combined weight of these two tilings is $\left(e^{i(n+1) \theta}-e^{-i(n+1) \theta}\right) / 2 i=\sin ((n+1) \theta)$, as desired.

More generally, as in Ericksen $(2008,2010)$, the same logic gives us:

Identity 5. For $n \geq 0$, and any real or complex number $z$,

$$
\left(z-\frac{1}{z}\right) U_{n}\left(\frac{z+\frac{1}{z}}{2}\right)=z^{n+1}-\frac{1}{z^{n+1}} \text {. }
$$

Identity 4 provides us with a composition theorem for Chebyshev polynomials of the second kind.

Identity 6. For $m, n \geq 0$,

$$
U_{m-1}\left(T_{n}(x)\right) U_{n-1}(x)=U_{m n-1}(x) .
$$

Proof. It suffices to prove this when $x=\cos \theta$. Here we have

$$
U_{m-1}\left(T_{n}(\cos \theta)\right) U_{n-1}(\cos \theta)=U_{m-1}(\cos (n \theta)) \frac{\sin (n \theta)}{\sin \theta}=\frac{\sin (m n \theta)}{\sin (n \theta)} \frac{\sin (n \theta)}{\sin \theta}=U_{m n-1}(\cos \theta) .
$$

\section{More trigonometric identities}

We conclude with two alternative expressions for $\cos (n \theta)$ and $\sin ((n+1) \theta)$ based on combinatorial arguments. First we prove a more general identity. 
Identity 7. For $n \geq 0$,

$$
T_{n}(x)=\sum_{k \geq 0}\left(\begin{array}{c}
n \\
2 k
\end{array}\right)\left(x^{2}-1\right)^{k} x^{n-2 k}
$$

Proof. For this proof, we use a slightly different combinatorial interpretation of $T_{n}(x)$, as given in Benjamin and Walton (2009), Benjamin and Walton (2010), and Walton (2007). Here we consider $n$-tilings with white squares, black squares, and dominoes with respective weights $x, x$, and -1 . But in order to accommodate the initial condition $T_{1}(x)=x$, the first cell must either be a domino or a white square. As usual, the weight of the tiling is the product of the weights of its tiles and $T_{n}(x)$ is the sum of the weights of all $n$-tilings.

Next observe that any such $n$-tiling can be decomposed in a unique way into "intervals of darkness" and "intervals of lightness," where an interval of darkness is either of the form $D b^{k}$ (a domino followed by $k$ black squares) where $k \geq 0$, or of the form $w b^{k}$ (a white square followed by $k$ black squares) where $k \geq 1$. Note that an interval of darkness must have length at least two. Everything outside an interval of darkness is necessarily a white square, and these comprise the intervals of lightness. For example, the tiling

\section{wwwwDDDDbbwbwwwbbbDwDbDw}

would decompose as

$w w w w D D D D b b w b w w w b b b D w D b D w$

where the intervals containing only $w$ are intervals of lightness, and the rest are intervals of darkness.

We claim that the summand gives the total weight of all $n$-tilings with exactly $k$ intervals of darkness. To see this, note that once you choose the endpoints for the $k$ intervals (which can be done in $\left(\begin{array}{c}n \\ 2 k\end{array}\right)$ ways), we can give the first two cells of the interval a weight of $x^{2}$ (for a white square followed by a black square) or a weight of -1 (for a domino). There is exactly one way to assign colors to the rest of the tiling, and those squares will contribute a weight of $x^{n-2 k}$.

Substituting $x=\cos \theta$ then gives the following identity (which has a simpler non-combinatorial proof, given in Chentzov et al., 1993).

Identity 8. For $n \geq 0$,

$$
\cos (n \theta)=\sum_{k \geq 0}\left(\begin{array}{c}
n \\
2 k
\end{array}\right)(-1)^{k}(\sin \theta)^{2 k}(\cos \theta)^{n-2 k}
$$

In a similar way, we can prove

Identity 9. For $n \geq 0$,

$$
U_{n}(x)=\sum_{k \geq 0}\left(\begin{array}{c}
n+1 \\
2 k+1
\end{array}\right)\left(x^{2}-1\right)^{k} x^{n-2 k} .
$$

Proof. Here $U_{n}(x)$ has the same interpretation as in the preceding proof, but we no longer have a restriction on the initial tile. This time, we choose $2 k+1$ points from the set $\{0,1, \ldots, n\}$. We begin the tiling with $j$ black squares where $j$ is the smallest chosen point $(j \geq 0)$. Then we proceed as in the $T_{n}$ identity with the remaining $2 k$ endpoints.

This time when we let $x=\cos \theta$ we get another identity from Chentzov et al. (1993).

Identity 10. For $n \geq 0$,

$$
\sin ((n+1) \theta)=\sum_{k \geq 0}\left(\begin{array}{c}
n+1 \\
2 k+1
\end{array}\right)(-1)^{k}(\sin \theta)^{2 k+1}(\cos \theta)^{n-2 k}
$$

\section{References}

Benjamin, A.T., Quinn, J.J., 2003. Proofs That Really Count: The Art of Combinatorial Proof. Mathematical Association of America, Washington, DC. Benjamin, A.T., Walton, D., 2009. Counting on Chebyshev polynomials. Mathematics Magazine 82, 117-126.

Benjamin, A.T., Walton, D., 2010. Combinatorially composing Chebyshev polynomials. Journal of Statistical Planning and Inference 140, $2161-2167$ [this issue].

Chentzov, N., Shklarsky, D., Yaglom, I., 1993. USSR Olympiad Problem Book. Dover Publications, Inc., New York.

Ericksen, L., 2008. Multiple product identities: balanced weights, thetas, finite gold. In: William, W. (Ed.), Applications of Fibonacci Numbers, vol. 11, Utilitas Mathematica Publishing Co., Winnipeg, to appear.

Ericksen, L., 2010. Lattice path combinatorics for multiple product identities. Journal of Statistical Planning and inference, this issue, doi:10.1016/j.jspi. 2010.01.017.

Shapiro, L.W., 1981. A combinatorial proof of a Chebyshev polynomial identity. Discrete Math. 34, 203-206.

Walton, D., 2007. A Tiling approach to Chebyshev polynomials. Senior Thesis, Harvey Mudd College, Claremont, CA 